\title{
New surrogate marker for ulcerative colitis (UC): PGE- major urinary metabolite (PGE-MUM)
}

\author{
Arihiro $\mathrm{S}^{1}$, Arai $\mathrm{Y}^{1}$, Matsuura $\mathrm{T}^{2}$, Okayasu $\mathrm{I}^{3}$, Ito $\mathrm{S}^{4}$ and Fujiwara $\mathrm{M}^{5 *}$ \\ ${ }^{1}$ Division of Gastroenterology and Hepatology, Department of Internal Medicine, The Jikei University Katsushika Medical Centre, Japan \\ ${ }^{2}$ Department of Laboratory Medicine, The Jikei University School of Medicine, Japan \\ ${ }^{3}$ Division of Nutrition, Kiryu University School of Health Care, Japan \\ ${ }^{4}$ IDAC Theranostics, Inc. Japan \\ ${ }^{5}$ Department of Pathology, Nissan Tamagawa Hospital, Japan
}

\section{Introduction}

Ulcerative colitis (UC) is an intractable bowel disease with an unknown etiology. Although long-term curable treatment is required for UC, remission is a practical goal of the current therapeutic approach. Thus, patients live in fear of relapse.

Remission is evaluated using colonoscopic and pathologic evaluation of the lower digestive tract. However, undergoing frequent colonoscopic observations for monitoring is stressful for patients even for efficacy assessments. Furthermore, biopsies are more stressful. Therefore, in routine clinical practice, annual colonoscopic and pathological analyses are performed during follow-up.

Recently new agents, especially biologics (e.g., anti-tumor necrosis factor- $\alpha$, anti- $\alpha 4 \beta 7$ integrin) and immune suppressors (JAK inhibitors) $[1,2]$ and new biomarkers have been introduced as new treatment options $[3,4]$. Therefore, we may set "mucosal healing" as the treatment goal for UC [5].

Diagnostics to monitor treatment efficacy involves the replaceability of pathological precision and is quantitative rather than qualitative in nature. The recent introduction of fecal calprotectin (produced by neutrophils) as a new biomarker for assessing UC stage [6] gives us a new option for assessing treatment efficacy. However, its qualitative nature and variable factors are drawbacks in discussions of the degrees of real mucosal healing [7-9].

Pathological assessment is qualitative and involves sampling probability. In Japan, dysplasia cases are subjected to targeted rather than randomized biopsies [10]. Therefore, around 6 points of biopsy samples are subjected to pathological observation. In the clinical consideration of remission, some points exhibit active scores in pathological assessments [11]. A long duration of UC may lead to fibrosis caused by periodical inflammation and remodelling with a high risk of cancer $[12,13]$. This is why we must help patients achieve remission at an early phase as soon as possible. Frequent colonoscopy and pathological biopsy assessments are stressful for patients; therefore, a new biomarker utilizing less stressful methods for sampling is needed. A new biomarker, prostaglandin E-major urinary metabolite (PGEMUM), was recently introduced to assess UC stage and activity [14]. This method is quantitative with daily reproducibility.

\section{PGE-MUM}

Prostaglandin E2 (PGE2) is a metabolite processed by cyclooxygenase-2, which is upregulated in inflammatory conditions. PGE2 has a short half-life in the blood; therefore, it may not be suitable for in-vitro diagnostic purposes $[15,16]$. However, its urinary metabolite (PGE-MUM) is stable and may be suitable for in vitro-diagnostics. Further, derivatizing into a bicyclic structure shows more stable characteristics for immunoassay recognized by specific antibodies to establish a radiolabelled immunoassay (RIA) [17]. Once the RIA system has been established, switching into the non-RIA style (enzyme-linked immunosorbent assay) is relatively easy. An automated system with a short assay time will also be introduced.

Because PGE2 is a biomarker of inflammation, the specificity of the inflamed organs is in question. However, only diseases with a wide range of inflammatory parts seem to reflect their inflammatory conditions on PGE-MUM. Thus, hepatitis and gastritis do not exhibit higher PGE-MUM values. Therefore, PGE-MUM is applicable for assessing UC activity evaluation in a practical fashion [18].

A report14 of the evaluation of adult UC showed that PGEMUM assessments of children's UC activity showed good correlations with clinical activity [19]. Once monitoring usability of PGE-MU is established, its value for medical practice may indeed be appreciated. In a future comparative study, the practical value of fecal calprotectin and/or PGE-MUM for UC patients will give us valuable information for further clinical practice.

Necrotic enterocolitis (NEC) is a rare disease of neonates that shows extremely high PGE-MUM values compared to those of UC cases. Whether any decision of an urgent surgical request can be made by setting the PGE-MUM value in NEC cases is now a big question. However, such information, if established, seems to provide essential clinical value. One such study is ongoing, and its preliminary results have been submitted for publication [K. Konishi et al., in press. J. Pediatr. Surg.].

*Correspondence to: Mutsunori Fujiwara, MD. PhD, Department of Pathology, Nissan Tamagawa Hospital, Japan, E-mail: m-fujiwara@mbg.ocn.ne.jp

Received: August 09, 2018; Accepted: August 23, 2018; Published: August 25, 2018 


\section{Conclusion}

After the diagnosis of UC is made, to determine whether PGEMUM is considered a real new biomarker to assess colitis activity and treatment monitoring clinically further discussions with evidence of clinical practice are needed. However, the value of PGE-MUM must be discussed. This fact is essential for urine sampling, which is least stressful for patients, especially children and infants/neonates. In pediatrics, general anaesthesia is a condition of a UC diagnosis, which carries risk. Therefore, the practical value of urine sampling to monitor UC treatment should be emphasized.

Replacing colonoscopic and pathological assessments with urinary measurements would be appreciated by adult patients. This will lead to better treatment follow-up to achieve mucosal healing with better quality of life and reduced medical costs.

\section{References}

1. Ungar B, Kopylov U (2016) Advances in the development of new biologics in inflammatory bowel disease. Ann Gastroenterol 29: 243-248. [Crossref]

2. Vickers AD, Ainsworth C, Mody R (2016) Systematic review with network metaanalysis: comparative efficacy of biologics in the treatment of moderately to severely active ulcerative colitis. PLoS One 11: e0165435. [Crossref]

3. Smith LA, Gaya DR (2012) Utility of faecal calprotectin analysis in adult inflammatory bowel disease. World J Gastroenterol 18: 6782-6789.

4. Takashima S, Kato J, Hiraoka S, Nakarai A, Takei D, et al. (2015) Evaluation of mucosal healing in ulcerative colitis by fecal calprotectin vs. fecal immunochemical test. Am J Gastroenterol 110: 873-880. [Crossref]

5. Walsh A, Palmer R, Travis S (2014) Mucosal healing as a target of therapy for colonic inflammatory bowel disease and methods to score disease activity. Gastrointest Endosc Clin N Am 24: 367-378.

6. Schoepfer AM, Beglinger C, Straumann A (2009) Ulcerative colitis: correlation of the rachmilewitz endoscopic activity index with fecal calprotectin, clinical activity, C-reactive protein, and blood leukocytes. Inflamm Bowel Dis 15: 1851-1858. [Crossref]

7. Toyonaga T, Kobayashi T, Nakano M (2017) Usefulness of fecal calprotectin for the early prediction of short-term outcomes of remission-induction treatments in ulcerative colitis in comparison with two-item patient-reported outcome. PLoS One 12: e0185131. [Crossref]
8. Calafat M, Cabre E, Manosa M (2015) High within-day variability of fecal calprotectin levels in patients with active ulcerative colitis: what is the best timing for stool sampling? Inflamm Bowel Dis 21:1072-6. [Crossref]

9. Lasson A, Stotzer PO, Ohman L (2015) The intra-individual variability of faecal calprotectin: a prospective study in patients with active ulcerative colitis. $J$ Crohns Colitis 9: 26-32.

10. Watanabe T, Ajioka Y, Mitsuyama K, Watanabe K, Hanai H, et al. (2016) Comparison of targeted vs random biopsies for surveillance of ulcerative colitis-associated colorectal cancer. Gastroenterology 151: 1122-1130. [Crossref]

11. Osada T, Ohkusa T, Okayasu I (2008) Correlations among total colonoscopic findings, clinical symptoms, and laboratory markers in ulcerative colitis. $J$ Gastroenterol Hepatol 2: 262-267. [Crossref]

12. Beaugerie L, Itzkowitz SH (2015) Cancers complicating inflammatory bowel disease. N Engl J Med 372: 1441-1452. [Crossref]

13. Okayasu I (2012) Development of ulcerative colitis and its associated colorectal neoplasia as a model of the organ-specific chronic inflammation-carcinoma sequence. Pathol Int 62: 368-280.

14. Arai Y, Matsuura T, Matsuura M, Fujiwara M, Okayasu I, et al. (2016) Prostaglandin E-Major Urinary Metabolite as a Biomarker for Inflammation in Ulcerative Colitis: Prostaglandins Revisited. Digestion 93: 32-39. [Crossref]

15. Goodwin JS, Ceuppens J (1983) Regulation of the immune response by prostaglandins. J Clin Immunol 3: 295-315. [Crossref]

16. Förstermann U, Neufang B (1983) Elimination from the circulation of cats of 6-ketoprostaglandin E1 compared with prostaglandins E2 and I2. J Pharm Pharmacol 35: 724-728. [Crossref]

17. Okayasu I, Ohnishi H, Sarandi I, (2014) Significant increase of prostaglandin E-major urinary metabolite in male smokers: a screening study of age and gender differences using a simple radioimmunoassay. J Clin Lab Anal 28: 32-41. [Crossref]

18. Arai Y, Arihiro S, Matsuura T (2014) Prostaglandin E-major urinary metabolite as a reliable surrogate marker for mucosal inflammation in ulcerative colitis. Inflamm Bowel Dis 20: 1208-16. [Crossref]

19. Hagiwara SI, Okayasu I, Fujiwara M (2017) Prostaglandin e-major urinary metabolite as a biomarker for pediatric ulcerative colitis activity. J Pediatr Gastroenterol Nutr 64 955-961. [Crossref]

Copyright: (C2018 Arihiro S. This is an open-access article distributed under the terms of the Creative Commons Attribution License, which permits unrestricted use, distribution, and reproduction in any medium, provided the original author and source are credited. 